\title{
Successful Cryo Electron Tomography of Vitreous Cryo Sections
}

\author{
Jason Pierson $^{1}$ and Peter J. Peters ${ }^{2}$ \\ 1. Materials \& Structural Analysis (formerly FEI), Thermo Fisher Scientific, Waltham, MA, USA. \\ 2. The Maastricht Multimodal Molecular Imaging institute, Maastricht, The Netherlands.
}

All life forms are governed by protein interactions that synergistically regulate, at the cellular systemslevel, the behavior of an organism. Elucidating or deciphering these complex macromolecular interaction networks, in a cellular context, is a central goal in all disciplines of modern biology. A staggering question remains, however, as to whether these complexes that are reconstructed, reconstituted, or isolated away from the cell reflect their natural, structural organization in living cells. Currently, one of the most suitable techniques for analyzing the spatial organization of macromolecular complexes within the cell is cryo electron tomography (cryo-ET) of vitreous cryo sections; [1] the other being cryo-ET of focused ion beam (FIB) milled lamellae [2].

Vitreous Cryo-Sectioning (VS) is an established technique that combines the power of both vitrification for immobilization (through high pressure freezing) and cryo-ultramictoromy for thinning large cells and tissues [3]. Its application to tomography, however, has been hindered by the procedure for creating proper attachment of the ribbons of VS to the support film of the EM grid. If the attachment is compromised, imaging is deterred. The ribbons of VS are lost during transfer to the microscope, irregular VS movement during image acquisition, and problems with placing individual tilted images into a common register. In addition, water contamination inside and around the microtome plagues the subsequent imaging of the VS. The second major hurdle is that the technique is prone to several cuttinginduced deformations [4], which include chatter, knife marks, crevassing, and compression. Of the four cutting-induced artifacts, compression is arguably the most worrisome [5]. This is mainly because the other artifacts can be controlled, if not eliminated, by proper specimen preparation and sectioning procedures.

Here we show the procedure of electrostatic charging in order to attach VS to an EM grid support film [6]. The ribbon of VS remains in place during transfer and storage and is devoid of stamping related artifacts. We have implemented a protective glove box surrounding the cryo-ultramicrotome that reduces the humidity around and within the microtome during sectioning. We also show cryo-ET reconstructions of VS of the $80 \mathrm{~S}$ ribosomes, both intracellular and in an isolated state that appear able to resist section-induced compression [7]. Our observations indicate that VS induced compression is nonuniform between whole cells that have been sectioned and their intracellular macromolecular complexes.

The technique of VS still remains a challenge; however the novel techniques presented here facilitate the procedure. We have shown that frost contamination is reduced inside the cryo ultramicrotome, when a protective anti-contamination glove box is used. We have also shown that electrostatic charging enhances attachment to an EM support film without compromising the integrity of ribbons of VS, so that the throughput of suitable areas for tomography can be increased compared to conventional methods. We obtained an initial glimpse of the putative $80 \mathrm{~S}$ ribosomal cellular structure, within thin VS, when these novel improvements were applied to yeast cells. We conclude that cryo-ET of VS, in spite of sectioninduced compression, is a suitable technique for charting the structural organization of cellular nanomachines, such as ribosomes, in a cellular environment. 


\section{References:}

[1] J Pierson et al, J. Electron Microsc. 60 (2011) p. 93-100.

[2] M Schaffer et al, Bio-protocol 5(17) (2015) e1575.

[3] A Al-Amoudi et al, EMBO J 23 (2004) p. 3583-8.

[4] A Al-Amoudi et al, J. Struct. Biol. 150 (2005) p. 109-121.

[5] A Rigort et al, J. Struct. Biol. 172 (2010) p. 169-179.

[6] J Pierson et al, J. Struct. Biol. 169 (2010) p. 219-25.

[7] J Pierson et al, J. Struct. Biol. 173 (2011) p. 345-9.

[8] The authors would like to acknowledge Helmut Gnaegi for significant contributions to this work.
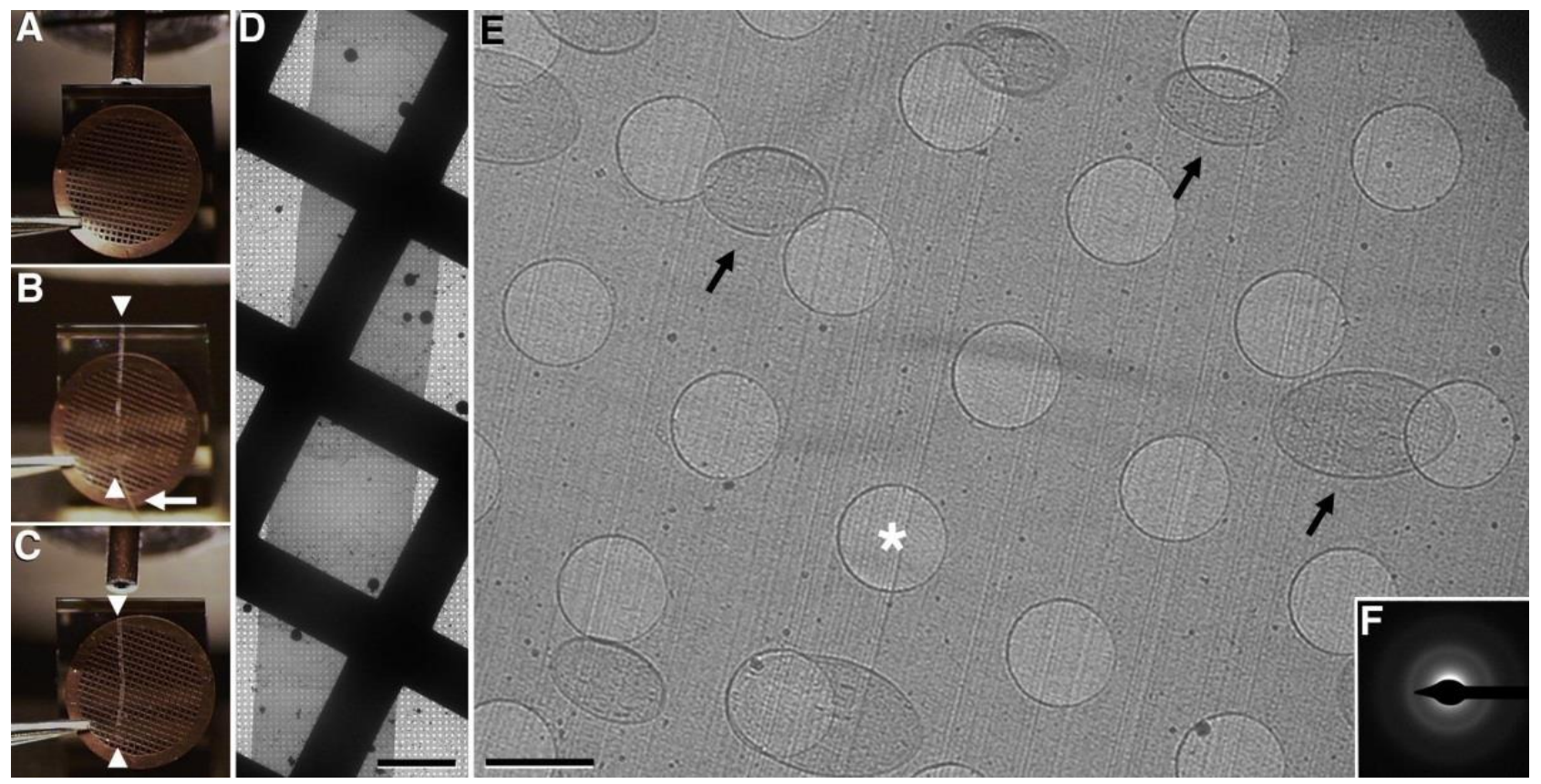

Figure 1. Electrostatic charging technique for attaching a ribbon of VS to an EM support film. (A-C) An EM grid is placed at the diamond knife-edge. A ribbon of VS (white arrowheads) is guided over the EM grid. Once the ribbon of VS is of suitable length the generator is switched from the discharge mode to the charge mode, causing the ribbon to attach to the EM grid. (D) A low magnification image showing the ribbon of VS after electrostatic charging. Scale bar $=50 \mathrm{~lm}$. (E) A medium magnification micrograph showing a VS from within the ribbon. Note that the section is smooth and relatively flat with no apparent mechanically induced damage as is often seen with stamping/pressing techniques. $S$.

cerevisiae cells can be seen scattered throughout the vitreous section (black arrows). Scale bar $=2 \mathrm{~lm}$. (F) A selected area diffraction pattern confirming vitreous water. 